\title{
Low-energy spectrum of an SU(2) gauge theory with dynamical fermions
}

\author{
Milan Vujinovic ${ }^{1,2, *}$ and Reinhard Alkofer ${ }^{1, \dagger}$ \\ ${ }^{1}$ Institute of Physics, NAWI Graz, University of Graz, Universitätsplatz, 5, 8010 Graz, Austria \\ ${ }^{2}$ Sao Carlos Institute of Physics, University of Sao Paulo, Avenida Trabalhador Saocarlense, \\ 400 Caixa Postal 369, CEP 13560-970 Sao Carlos, Brazil
}

(Received 12 September 2018; published 29 November 2018)

\begin{abstract}
The spectrum of light bound states in an SU(2) gauge theory with two flavors of fundamentally charged fermions is investigated by solving the Bethe-Salpeter equations in the respective channels within a 3PI-type (i.e., beyond rainbow-ladder) truncation including, self-consistently, a correspondingly truncated fermion-gauge boson vertex. Remarkable differences with respect to the meson spectrum of an SU(3) gauge theory are found; although, in our approach, these are not as pronounced as indicated by some recent respective investigations within lattice gauge field theory.
\end{abstract}

DOI: $10.1103 /$ PhysRevD.98.095030

\section{INTRODUCTION}

Understanding the physics of bound states in generic gauge field theories is for several reasons of interest. Over the last decades great efforts have been undertaken to describe hadrons as bound states of quarks and glue within QCD. Certain types of beyond-standard-model (BSM) approaches as composite Higgs models [1,2] and technicolor theories $[3,4]$ also require us to understand the physical spectrum of quantum field theories, including those of the strongly interacting type. Last but not least, dark matter might occur in the form of bound states within a hidden strongly interacting sector; cf. the so-called SIMP scenario [5].

Only recently the necessary tools for studying bound states in strongly interacting quantum field theories have been developed. On the one hand, in lattice gauge theories (hadron) spectroscopy has proven to be a much more complicated task than previously expected; see, e.g., Ref. [6] and references therein. On the other hand, due to the rich and quite often complicated structure of highly relativistic bound states of elementary constituents with spin, studies of hadrons within functional methods have been restricted to generalized rainbow-ladder (RL) truncations of the Bethe-Salpeter equation until some years ago; see, e.g., Ref. [7] and references therein, and even nowadays most of such investigations of bound-state properties

\footnotetext{
*milan.vujinovic@ifsc.usp.br

reinhard.alkofer@uni-graz.at
}

Published by the American Physical Society under the terms of the Creative Commons Attribution 4.0 International license. Further distribution of this work must maintain attribution to the author(s) and the published article's title, journal citation, and DOI. Funded by SCOAP. like, e.g., form factors and decays, still rely on RL-type approximations together with a phenomenologically adapted momentum dependence of the constituents' interactions.

The description of relativistic bound states from quantum field theory dates back to the seminal papers by Bethe and Salpeter [8]. Whereas their treatment of the deuteron was based on an expansion of the kernel, modern functional methods emphasize the importance of symmetries; for reviews on either the framework of Dyson-Schwinger $[9,10]$ and Bethe-Salpeter equations [8] or the functional renormalization group [11,12], see [13-18]. Within the Dyson-Schwinger-Bethe-Salpeter framework, the simplest symmetry-preserving truncation scheme is given by keeping the sum of all rainbow diagrams in the one-particle selfenergy, (i.e., in the two-point function) and all ladder diagrams in the four-point function. This RL truncation is the simplest scheme which obeys the constraints from the axial-vector Ward-Takahashi identity. This is an important feature in QCD as it guarantees the Goldstone boson nature of the pion. However, despite its considerable successes in the phenomenological studies of mesons and baryons, this and related truncation schemes have some serious practical and conceptual limitations; see, e.g., [7,19-38] for the successes and shortcomimgs of RL-type truncations for mesons and baryons.

Therefore, one of the long-standing goals within functional methods is to establish more sophisticated truncation schemes that can be systematically applied to reliably calculate bound-state properties. This task can be approached in two different ways: bottom-up or top-down. While the former uses phenomenological input in order to construct models and determine their parameters, the latter requires a robust theoretical foundation upon which to build. Consequently, there is a rich and diverse history regarding truncations of relativistic bound state equations. 
(N. B.: BRL truncations can be roughly categorized as diagrammatic; see, e.g., [39-53], and for nondiagrammatic approaches, see, e.g., [54-60].) Recent investigations have proven that it is essential to solve, at least, for the threepoint vertices of the elementary constituents explicitly in an (at least approximately) self-consistent procedure. In Ref. [53], light mesons have been investigated in what is, thus far, the most sophisticated truncation scheme to QCD within the Dyson-Schwinger-Bethe-Salpeter framework. Based on the three-particle irreducible effective action quark-loop contributions to the gluon propagator and three-gluon vertex have been taken into account. The resulting fully coupled system of Dyson-Schwinger equations for two- and three-point functions have been solved self-consistently. The symmetry-preserving quark-antiquark kernel of the Bethe-Salpeter equation for mesons has been derived, and timelike properties of bound states have been obtained by analytic continuation of Euclidean momenta.

Related studies of QCD have been performed recently with functional renormalization group techniques; see Ref. [61] and references therein. Employing a vertex expansion scheme based on gauge-invariant operators a quantitative analysis of chiral symmetry breaking has been performed, and the feasibility of dynamical hadronization has been demonstrated. The resulting quark propagator, quark-gluon vertex (including its full tensor structure and momentum dependence), and some properties of the fourfermion scattering kernels have been calculated. However, the analytic continuation necessary to discuss bound states could not be achieved yet. This shortcoming in mainly due to the lack of suitable regulator functions which are a defining element of the functional renormalization group equations. Recent progress on this issue [62] demonstrates the technical nature of this limitation and makes evident that it will be overcome in the near future.

In recent years, $\mathrm{SU}(2)$ gauge theories have been studied mainly for two reasons. Two-color gauge theories with an even number of fermion flavors $N_{f}$ have been of interest to lattice practitioners because Monte Carlo lattice simulations of them at nonvanishing chemical potential are not hindered by the sign problem, and one can gather information about the phase diagram of the corresponding strongly interacting matter on the lattice [63-70]. This then also initiated related studies with functional methods $[50,71,72]$. Recently, an $S U(2)$ gauge theory with two fundamentally charged Dirac fermions has been studied on the lattice [73-77] because it provides the simplest field theoretical realization of a unified theory of a composite Goldstone boson Higgs and technicolor [78]. Therefore, such a theory might serve as a template for aspects of dynamical electroweak symmetry breaking as well as the SIMP scenario for dark matter.

In this investigation, light fermion-anti-fermion ("mesons") and fermion-fermion (bosonic two-color "baryons") bound states are studied in an $\mathrm{SU}(2)$ gauge theory with two fundamentally charged fermions within a beyond-rainbowladder (BRL) truncation to the respective Dyson-Schwinger (DS) and Bethe-Salpeter (BS) equations. A certain focus is given on an analysis of the impact of various diagrammatic contributions to the fermion-gauge boson vertex function on bound-state observables. Hereby suitable model input for the Yang-Mills two- and three-point functions is used to evaluate the fermion-gauge boson vertex in a semi-selfconsistent way. While there are similar calculations available [50,51], the present study improves upon these in the truncation for the quark-gluon-vertex DS equation; for details, see Sec. II B.

The paper is organized as follows: In Sec. II, the employed bound-state equations as well as the determination of the necessary input are provided and discussed, especially also with respect to preserving chiral symmetry. In Sec. II B, the used truncations for the equation of the fermion-gauge boson vertex are introduced. These constitute the main element of the BRL truncation utilized in the following, and therefore it is discussed how different elements of the coupled system of DS equations for the fermion-gauge boson vertex and the fermion propagator influence this fundamental vertex function. In Sec. IIE, a symmetry-preserving kernel of the bound state equation is presented, and in Sec. III, the spectrum of light fermionantifermion and fermion-fermion bound states in a $S U(2)$ gauge theory with two fundamentally charged fermions is presented. In Sec. IV, our conclusions are provided. Some technical issues related to solving for the fermion-gauge boson vertex are deferred to the Appendix.

\section{THE BOUND-STATE EQUATION}

\section{A. Constraining the kernel}

In an SU(2) gauge theory, mesonic-type and baryonictype bound states are both two-body bound states and bosons; their respective channels are related by the socalled Pauli-Gürsey symmetry and are, therefore, degenerate. In the following, it is therefore completely sufficient to focus on the fermion-antifermion bound states to understand the low-lying spectrum. Nevertheless, degeneracy factors of the multiplets have to take into the account the existence of fermion-fermion (baryonic-type) bound states.

Understanding the quantum numbers of possible Goldstone bosons which might appear in the chiral limit will provide us some guiding principle when choosing a truncation to the bound state equation; see below the discussion of the axial-vector Ward-Takahashi identity (axWTI). The pseudoreality of the fundamental representation of the group SU(2) implies also that the flavor symmetry is upgraded to SU(4). Dynamical chiral symmetry breaking leaves a $\mathrm{Sp}(4)$ [locally isomorphic to $\mathrm{SO}(5)]$ intact, and therefore one expects to have in the chiral limit five Goldstone bosons: three of them are fermion-antifermion bound states (similarly to the pions 
in QCD), and two of them are of the baryon and, respectively, diquark type. Note that, in the latter case, the Pauli principle reduces the number of states; cf. e.g., the discussion in Ref. [79] and references therein. Note, also, that a meson-type state with $J^{P}$ quantum numbers will have the same mass as a diquark-type state with equal total spin and opposite parity $\left(J^{-P}\right)$. Thus, with the meson spectrum obtained, one can immediately extend the results and conclusions to the two-fermion states as well.

Using this setup as a model for electroweak symmetry breaking, the "direction" of the symmetry breaking with respect to standard model determines the nature of the model: For a vanishing angle one obtains a composite Higgs model where four of the five Goldstone bosons provide the Higgs doublet, and the fifth is neutral under standard model charges. At the maximal angle one obtains a technicolor theory. Three of the Goldstone bosons enter as members of BRST quartets, and thus the longitudinal components of the $W$ and $Z$ bosons become physical. The Higgs is then the lightest scalar bound state, and the remaining two Goldstone bosons can be considered as dark matter candidates. In case the angle is determined dynamically to be neither vanishing nor maximal, the Goldstone boson Higgs mixes with the technicolor scalar bound state, and the lighter of the two scalars will be identified with the physical Higgs. Needless to say that then the spectrum of this theory will be more complicated to understand than in the two extreme cases. Last but not least, the back-coupling to standard model particles will lead to large corrections (see e.g., [80]), and therefore some quite elaborate studies are required before one can judge the usefulness of such a theory as a beyond-the-standard-model scenario.

The two-body BS equation, depicted in Fig. 1, for the amplitude $\Gamma_{M}(p, P)$ takes generically the form (see, e.g., [14])

$$
\left[\Gamma_{M}(p, P)\right]_{i j}=\int_{k}[K(p, k, P)]_{i k ; l j}\left[\chi_{M}(k, P)\right]_{k l} .
$$

Hereby, $P$ denotes the total four-momentum of the bound state, and $p$ the relative momentum between the constituents. It is implicitly understood that the amplitude is projected onto an eigenstate of the Pauli-Lubanski vector and on parity and charge conjugation eigenstates; i.e., $J^{P C}$ are good quantum numbers. The abbreviation $\int_{k}$ stands for $\int d^{4} k /(2 \pi)^{4}$, and $K(p, k, P)$ denotes the interaction kernel.

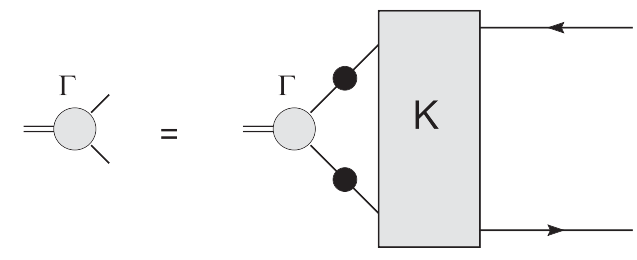

FIG. 1. The meson Bethe-Salpeter equation.
$\chi_{M}(k, P)$ is the so-called $\mathrm{BS}$ wavefunction which is related to amplitude via the relation

$$
\chi_{M}(k, P)=S\left(k_{+}\right) \Gamma_{M}(k, P) S\left(k_{-}\right)
$$

with $S\left(k_{ \pm}\right)$being the fermion propagator. Although there is some freedom in the momentum assignement we choose the momenta $k_{ \pm}$to be $k_{ \pm}=k \pm P / 2$ which is the optimal choice when solving Eq. (1) numerically.

An essential ingredient needed for the evaluation of the BS equations is the fermion propagator $S(k)$. In covariant gauges, it can be decomposed as

$$
S^{-1}(p)=Z_{f}^{-1}\left(p^{2}\right)\left[i \not p+M\left(p^{2}\right)\right],
$$

where $Z_{f}\left(p^{2}\right)$ is the respective fermion wavefunction renormalization, and $M\left(p^{2}\right)$ is a dynamically generated mass function. At tree level, the above expression simplifies to $S_{0}^{-1}(p)=i \not p+Z_{m} m$, with $Z_{m}$ being the fermion mass renormalization constant. The fermion two-point function satisfies its own Dyson-Schwinger equation, given by (see also Fig. 2)

$$
\begin{aligned}
S^{-1}(p)= & Z_{2} S_{0}^{-1}(p) \\
& +g^{2} Z_{1 f} C_{f} \int_{k} \gamma^{\mu} S(k+p) \Gamma^{\nu}(k+p, p) D_{\mu \nu}(k) .
\end{aligned}
$$

The functions $D_{\mu \nu}(k)$ and $\Gamma^{\nu}(k+p, p)$ are, respectively, the full gauge-boson propagator and the fermion-gauge boson vertex. $C_{f}$ is the gauge group's Casimir invariant in the fundamental representation, with $C_{f}=3 / 4$ in an $S U(2)$ gauge theory. $Z_{2}$ and $Z_{1 f}$ are the renormalization constants for the fermion field and the fermion-gauge boson three-point interaction, respectively. In the next section, we provide details on how the various renormalization factors are obtained.

The four-point kernel $K(p, k, P)$ of Eq. (1) subsumes an infinity of processes through which a fermion and an antifermion can interact. Obviously, any practical consideration of the BS equation requires the interaction kernel to be truncated. In the studies of light-light and heavy-light mesons, an important guideline for these truncations has been and is provided by the axial-vector Ward-Takahashi identity (axWTI), which connects the respective four-point function $K$ to the quark self-energy $\Sigma(k)$ of Eq. (4):

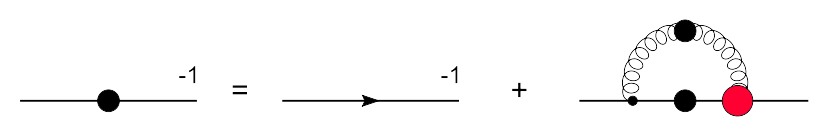

FIG. 2. The DS equation for the fermion propagator. Straight lines are quarks, wiggly ones gluons. Filled circles denote full propagators and vertices. 


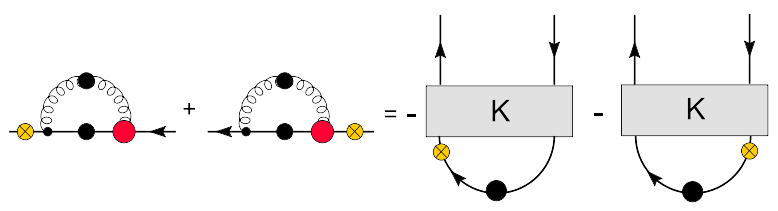

FIG. 3. A diagrammatic form of the axWTI for flavor nonsinglet mesons. Yellow blob stands for the $\gamma_{5}$ matrix.

$$
\begin{aligned}
& {\left[\Sigma\left(p_{+}\right) \gamma_{5}+\gamma_{5} \Sigma\left(p_{-}\right)\right]_{i j}} \\
& \quad=\int_{k}[K(p, k, P)]_{i k ; j}\left[\Sigma\left(k_{+}\right) \gamma_{5}+\gamma_{5} \Sigma\left(k_{-}\right)\right]_{k l} .
\end{aligned}
$$

A diagrammatic representation of the axWTI for flavor nonsinglet mesons is given in Fig. 3. If a particular truncation for the quark DS and meson BS equation satisfies this identity, the special status of light pseudoscalar mesons as (pseudo-) Nambu-Goldstone bosons will remain intact, and the masslessness of the pseudoscalar ground states in the exact chiral limit is guaranteed. As it is expected that chiral symmetry and its breaking patterns play an important role also in technicolor and/or composite Higgs models, we require in the following the axWTIinduced relation between the kernels of the BS and the DS equations.

One way to obtain an axWTI-preserving kernel $K$ from an approximated fermion DS equation is to require the kernel $K$ in coordinate-space to be given by the following functional derivative of the fermion's self-energy $\Sigma$ with respect to the fermion's propagator (also in coordinatespace)

$$
K\left(x_{1}, x_{2}, x_{3}, x_{4}\right)=-\frac{\delta \Sigma\left(x_{1}, x_{2}\right)}{\delta S\left(x_{3}, x_{4}\right)} .
$$

In a diagrammatic language, the operation of Eq. (6) corresponds to "cutting" all internal fermion lines in the fermion propagator DS equation to generate the kernel of the $\mathrm{BS}$ equation $[39,40]$. An illustration is provided by the simplest nontrivial scheme which obeys the axWTI, the RL truncation. Starting by replacing the fully dressed fermiongauge boson vertex in Eq. (4) with its tree-level counterpart, possibly multiplied by a function of the gauge-boson's momentum squared, $\lambda\left(k^{2}\right)$, i.e.,

$$
\Gamma^{\nu}(k+p, p) \rightarrow \lambda\left(k^{2}\right) \gamma^{\nu},
$$

one applies the above described cutting technique. It is then straightforward to derive that the corresponding symmetrypreserving BS kernel is given by an exchange of a single dressed gauge boson which is shown in Fig. 4 and which consitutes one rung of the "ladder" generated by iteration of the kernel within the BS equation. In most of its hadron physics applications, the function $\lambda\left(k^{2}\right)$ of Eq. (7) was combined with the nonperturbative dressing of the gluon

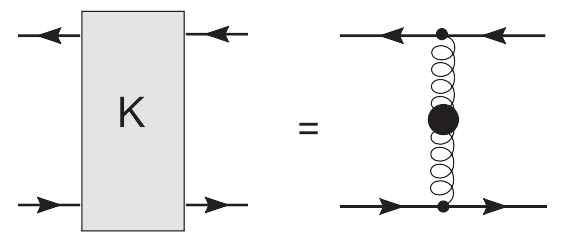

FIG. 4. The ladder truncation of the meson BSE interaction kernel.

propagator into a single effective interaction, and the model parameters are chosen such that some hadronic observables (e.g., the pion decay constant $f_{\pi}$ and the $\rho$ vector meson mass $m_{\rho}$ ) are correctly reproduced.

As mentioned in the introduction the BS approach in RL truncation has enjoyed considerable successes in the phenomenological studies of hadrons, and it is still widely used today. Of course, a major part of this success is related to choosing the interaction model parameters by fitting to a few hadronic observables. To which extent the function $\lambda\left(k^{2}\right)$ reflects properties of the quark-gluon vertex stays elusive. Since the whole formalism in the RL truncation is reflecting basically only the properties of the calculated quark propagator, it is in general not possible to disentangle the various physical processes and interactions (like, e.g., pion cloud effects) which contribute to measurable quantities [81,82], or to assess the influence of gauge degrees of freedom. Additionally, it is virtually impossible to adjust the RL framework to strongly interacting theories different from QCD, since there is no obvious and meaningful way to reparametrize the effective interaction(s) to account for changes in the principal vertex functions of the model; see, e.g., [50]. Furthermore, in a solution for the coupled DS equations for the QCD propagators with a rainbow truncation for the quark DS equation the onset of the conformal window occurs for a too small number of quark flavors [83]. This can be related to overestimating the quark loop in the gluon DS equation, and thus can only be improved if more than the tree-level structures of the quark-gluon vertex are taken into account. This implies then for the current investigation to use a more general framework including especially solving, at least approximately, for the fermiongauge boson three-point vertex.

\section{B. The fermion-gauge boson three-point vertex}

The three-point fermion-antifermion-gauge boson vertex possesses in covariant gauges in general twelve tensor components. In Landau gauge, only those eight components are needed which are purely transverse to the gaugeboson's momentum. Therefore, and also because other needed correlation functions are best known in this gauge, the investigation reported herein is done within the Landau gauge.

The DS equation for the three-point fermion-gauge boson vertex equation can be written in two different 


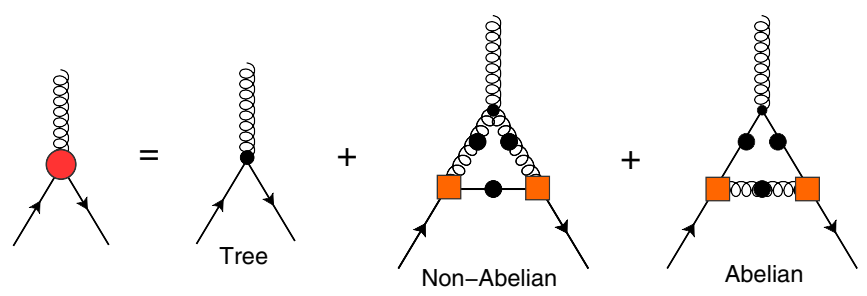

FIG. 5. The truncated fermion-gauge boson three-point vertex DS equation, in "1PI" formulation. The internal fermion-gauge boson vertices (orange squares) are modeled; see Appendix for details. We also consider the "3PI" approximation, with all internal vertices dressed.

forms. These as well as the corresponding derivations may be found, e.g., in Ref. [84], chapter 2 of Ref. [85], or chapter 7 of Ref. [86]. For the sake of brevity, we focus immediately on the truncated form shown in Fig. 5. The truncation (see Refs. [49,53,84-86] for its justification) consists of considering the one-loop contributions which contain primitively divergent vertices. These then retain two diagrams on the rhs of the truncated DS equation which are usually labeled as the non-Abelian and Abelian diagrams. Retaining one or both of these two contributions is the usual approximation when treating the quark-gluon vertex function in functional or semiperturbative approaches; see, e.g., Refs. [47,49,53,81,84-90] (but also Refs. [91-94] for significantly different continuum formulations).

As will become evident below it is not necessary to treat this three-point vertex function in a fully self-consistent way: Instead of back-coupling the full vertex function (the red blob in Fig. 5) into its DS equation, a projected version is used for the internal vertices (denoted by orange squares in Fig. 5). As this point is of a completely technical nature all details of this procedure are described in Appendix. It is sufficient to note for the following that for the full vertex the complete eight transverse components are used. For the purpose of solving its DS equation, however, we project it onto the respective tree-level component, thereby calculating an effective dressing $\lambda\left(k^{2}\right)$, and in turn we employ this on the rhs of the vertex DS equation.

In the context of bound state studies, the above-mentioned approximation provides a significant and almost necessary technical simplification. The most important reason for this approximation relates to the implementation of the cutting procedure for the construction of a symmetrypreserving BS kernel. If one were to employ the vertex in a fully self-consistent way one would have to take into account its implicit fermion propagator dependence, and the functional derivative in Eq. (6) would produce some very complicated terms in the bound state equation as, e.g., a five-point Green function with four fermion and one gauge boson leg. While it is possible to obtain a solvable BS kernel with a self-consistent treatment of the fermiongauge boson vertex (see, e.g., Refs. [52,53]), so far no one has tackled the challenge of solving the bound-state equation with the Abelian loops included in a selfconsistent manner. Our calculation can thus be seen as an intermediate step towards a more complete treatment: Additional diagrams are included, both in the vertex equation and the BS kernel, but the evaluation of the fermion-gauge boson vertex itself is considerably simplified without loosing, at least partially, the back-coupling effect of the vertex on itself.

Besides the one-particle irreducible (1PI) equation, depicted in Fig. 5, we also consider a form derived from the three-particle irreducible (3PI) formalism [95]; cf. also Refs. $[53,85,86,88]$, with all the internal vertices dressed. The notation 1PI/3PI should hereby not be understood in a strict manner but more as convenient labels for the presentation of the results, mainly because 1PI or 3PI formulations would entail a self-consistent evaluation of all vertex functions. Note that additional vertex dressings in the 3PI approach can effectively be seen as a partial inclusion of the disregarded two-loop terms. In addition, they also provide an estimate of the truncation errors. For the non-Abelian diagram in the 3PI framework also the fully dressed three-gauge boson vertex is required.

\section{Gauge boson correlation functions}

As became evident in the discussion above, the gaugeboson propagator and the three-gauge boson vertex are needed as input. For determining the latter, one also the ghost propagator will serve as input. In Landau gauge, the gluon and ghost propagators, $D_{\mu \nu}(k)$ and $D_{G}(k)$, respectively, are of the form

$$
D_{\mu \nu}(k)=T_{\mu \nu}^{(k)} \frac{Z\left(k^{2}\right)}{k^{2}}, \quad D_{G}(k)=-\frac{G\left(k^{2}\right)}{k^{2}},
$$

with $T_{\mu \nu}^{(k)}=\delta_{\mu \nu}-k_{\mu} k_{\nu} / k^{2}$ being the transverse projector with respect to momentum $k$. The dressing functions $Z\left(k^{2}\right)$ and $G\left(k^{2}\right)$ can be determined from their respective DS equations. A compilation of results for Yang-Mills correlation functions at different levels of truncations can be found in Ref. [96]. An example for those dressing functions (which are then used also in the following) are shown in Fig. 6. Details of their calculation are given in Ref. [97]. Obtaining the Yang-Mills propagators from their DS equations has the benefit of providing not only the required dressing functions but also other essential input such as renormalization constants. Hereby, $\tilde{Z}_{3}$ and $Z_{3}$ being, respectively, the ghost and gauge boson renormalization constants are used to determine the corresponding renormalization constants for other Greens functions via SlavnovTaylor identities. We will return to this point below when we will discuss the numerical method for the coupled system of DS equations for the fermion propagator and the fermion-gauge boson vertex.

For the three-gauge boson correlation function we use the truncation depicted in Fig. 7. First of all, based on the 


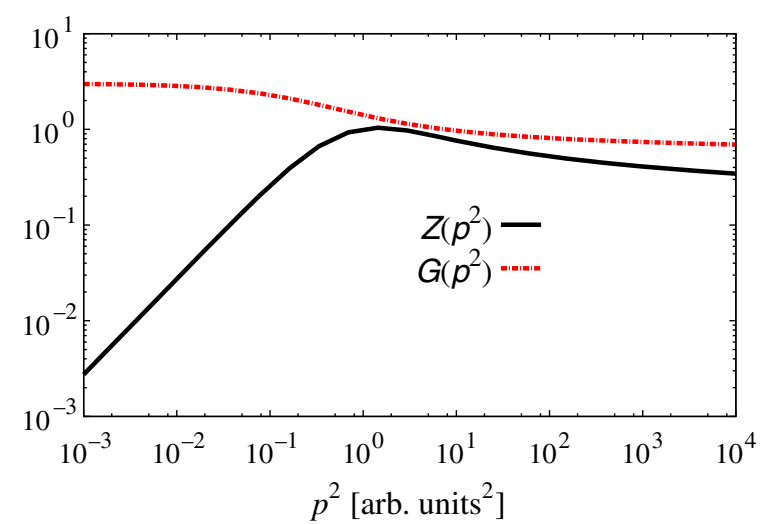

FIG. 6. Model dressing functions for ghost $(G)$ and gauge boson $(Z)$ propagators as function of the square of an arbitrary momentum scale. The connection to physical scales is established in Sec. III.
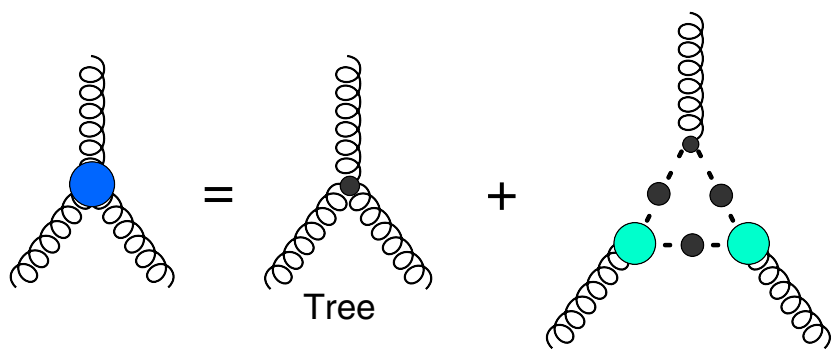

Ghost triangle

FIG. 7. A truncated DS equation for the three-gauge boson vertex. The full ghost-gauge boson vertices are approximated by bare ones.

results of Ref. [97] it is a fair approximation to keep for the gauge group and Lorentz tensor structure only the tree-level ones. Hereby, the tree-level three-gauge boson vertex is denoted by $\Gamma_{\mu \nu \rho}^{(0)}\left(p_{1}, p_{2}, p_{3}\right)$. Second, the momentum dependence of the multiplying function can be quite well represented by the form

$$
\Gamma_{\mu \nu \rho}\left(p_{1}, p_{2}, p_{3}\right)=\mathcal{A}\left(s_{0}\right) \cdot \Gamma_{\mu \nu \rho}^{(0)}\left(p_{1}, p_{2}, p_{3}\right),
$$

where the function $\mathcal{A}$ depends only on the symmetric momentum variable $s_{0}=(1 / 6) \cdot\left(p_{1}^{2}+p_{2}^{2}+p_{3}^{2}\right)$. The model dressing function $\mathcal{A}$ is taken from a DS calculation following Ref. [97]. However, here we do not include all of the self-energy contributions which were considered in this reference but instead choose a "ghost-loop-only" approximation as depicted in Fig. 7. The resulting dressing function is shown in Fig. 8. Note that these restrictions, in terms of the employed tensor structures, the momentum dependence and the only kept diagram, are well justified by previous results on the three-gluon vertex $[53,97,98]$ which in turn are substantiated by lattice results [99] although in four dimensions they are somewhat inconclusive at lower

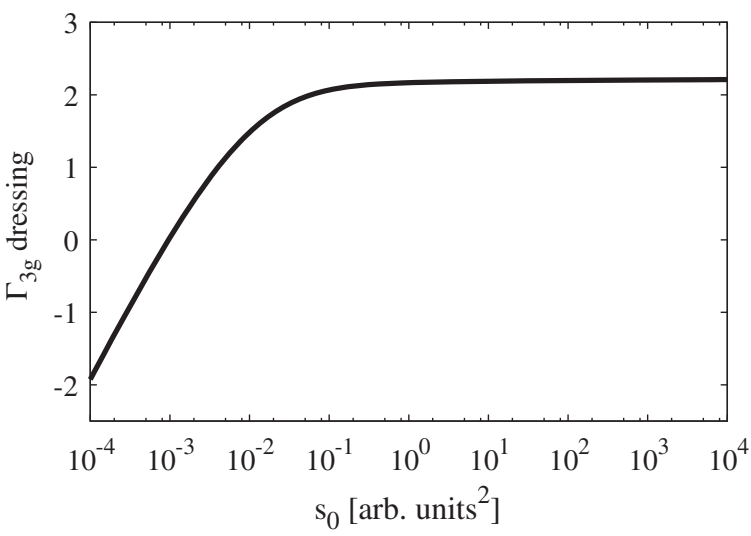

FIG. 8. Dressing for the three-gauge boson vertex as a function of the momentum variable $s_{0}=(1 / 6) \cdot\left(p_{1}^{2}+p_{2}^{2}+p_{3}^{2}\right)$ in arbitrary units. Connection to physical scales is established in Sec. III.

energies due to the large statistical uncertainties. (N. B.: For a discussion of the technical difficulties to extract the threegluon vertex from lattice gauge-field configurations, see Ref. [100].)

Here a remark is in order: All Yang-Mills input is taken from DS calculations which were originally performed for QCD, i.e., for an SU(3) gauge theory. Nevertheless, these functions can equally serve as input into our SU(2) calculation without any changes due to the choice of truncating the DS equations. In the ghost and gluon propagator as well as the three-gluon vertex computations specified in Ref. [97], only those diagrams were retained which are proportional to the product $g^{2} N_{c}$, with $g$ the gauge coupling and $N_{c}$ the number of colors. Thus one can easily account for the difference in the number of colors by changing the renormalization condition for the running coupling accordingly. The product $g^{2} N_{c}$ remains the same as in QCD, and all DS equations are formally remain unchanged. This simple trick would have been impossible if, e.g., the unquenching effects were taken into account for either of the vertex functions.

\section{Numerical results for the fermion propagator and fermion-gauge boson vertex}

With the Yang-Mills input specified, we briefly comment on the solution method for the coupled set of equations for the fermion propagator and fermion-gauge boson vertex. We use a fixed-point iteration technique, starting with an initial guess for the fermion dressing functions and the respective field renormalization constant $Z_{2}$. Note that in the chiral limit a single renormalization condition for fermions is sufficient. In addition, this quantity is ultraviolet finite in the Landau gauge. From $Z_{2}$ and the ghost propagator input the fermion-gauge boson vertex renormalization constant $Z_{1 f}$ is determined from a simple identity which is valid in the mini-MOM scheme [101] in Landau 

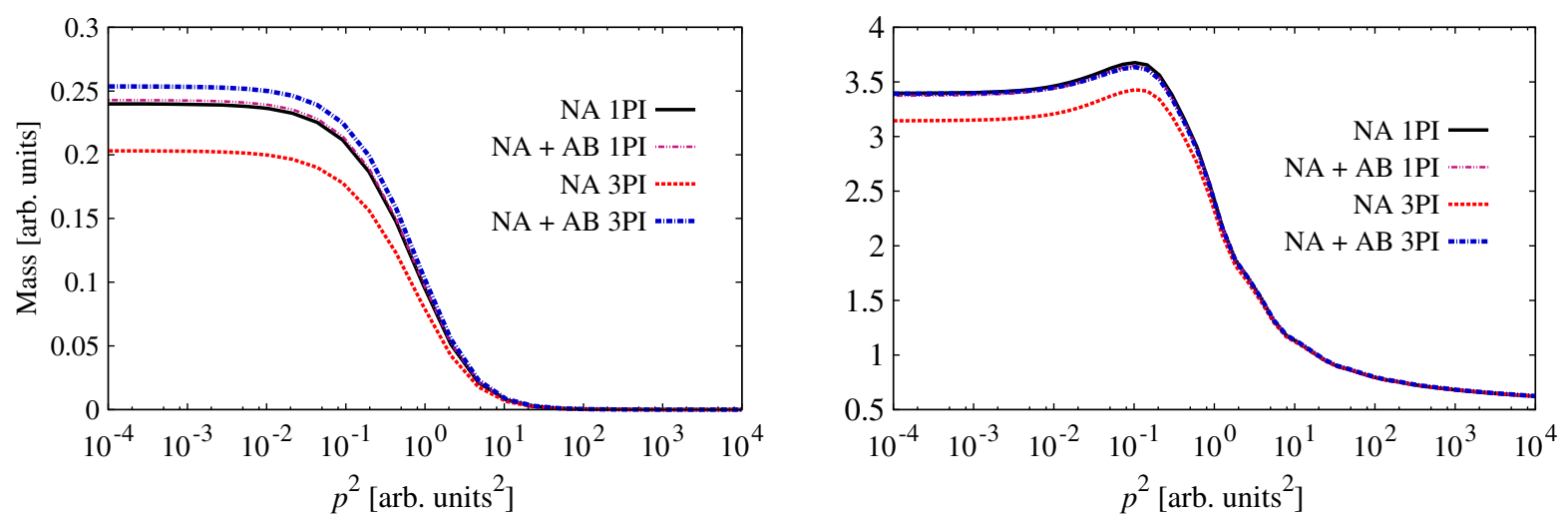

FIG. 9. The dynamically generated fermion mass function (left) and the fermion-gauge boson tree-level tensor structure $T_{1}\left(p^{2}, 2 p^{2}, 3 p^{2}\right)$ (right) in different truncations for the fermion-gauge boson DS equation. "NA" labels the calculation with the non-Abelian diagram only and "AB" includes the Abelian one. The labels 1PI/3PI are explained in the text and in the caption of Fig. 5.

gauge: $Z_{1 f}=Z_{2} / \tilde{Z}_{3}$. As in this work we will report only on results in the chiral limit, this fixes all the ingredients needed in the vertex DS equation. Therefore, the vertex can be evaluated and back-fed into the quark propagator DS equation until convergence is reached. All further details, and especially how the internal vertex is obtained from treelevel projection of the fully calculated vertex, are delegated to Appendix.

In Fig. 9, the corresponding results for the fermion mass function and the dominant (tree-level) tensor structure of the fermion-gauge boson vertex are displayed for several truncations. As noted before, we consider both the 1PI- and 3PI-type of DS equations, and we also study a truncation in which only the non-Abelian diagram (NA) has been retained in the vertex equation in order to probe the relative strengths of various contributions. One of the first things to note is that in the 1PI-based truncation the influence of the Abelian diagram is virtually nonexistent compared to the non-Abelian one. This is in accordance with previous results for the quark-gluon [81] and scalar-gluon [102] vertex functions. However, we note already here that despite its negligible effect on these fundamental vertex functions, the Abelian diagram induces a moderate correction for meson masses in 1PI formulation as will be seen in the next section. It would, thus, not be entirely correct to assume that these diagrams can be completely neglected in the 1PI approach, at least when bound state studies are concerned.

By considering the results with the non-Abelian diagram alone, one can see that the dressed three-gauge boson vertex has an appreciable impact leading to a significant reduction in the dressing functions. The screening effects of the full gauge-boson three-point correlation function in the 3PI-based approach are almost canceled exactly by the dressed third fermion-gauge boson vertex in the Abelian diagram. Due to this cancelation (which may or may not be coincidental) the final results are almost identical in the 1PI- and 3PI-based approaches.
A further test of how close the results for the fermion propagator are in these two different approaches is provided by its spectral functions. To this end, we calculate the fermion scalar spectral function by Fourier transforming $\sigma_{S}\left(p^{2}\right)=Z_{f}\left(p^{2}\right) M\left(p^{2}\right) /\left(p^{2}+M^{2}\left(p^{2}\right)\right):$

$$
\sigma_{S}(t)=\int d^{3} x \int \frac{d^{4} p}{(2 \pi)^{4}} e^{i p \cdot x} \sigma_{S}\left(p^{2}\right)
$$

In Fig. 10, the absolute values of $\sigma_{S}(t)$ in both approaches are displayed. The cusps in the curves correspond to zero crossings of $\sigma_{S}(t)$ and signal positivity violation for the fermions. First of all, we note again the close proximity of the results for the two different approaches.

With respect to the inferred positivity violation, a remark is in order here. Patterns similar to the one depicted in Fig. 10 were found also in some simpler truncation schemes, e.g., in the rainbow approximation to the quark propagator DS equation; cf. Ref. [103] and references therein. However, the investigation reported in [103] provided hints that the quark positivity violation within

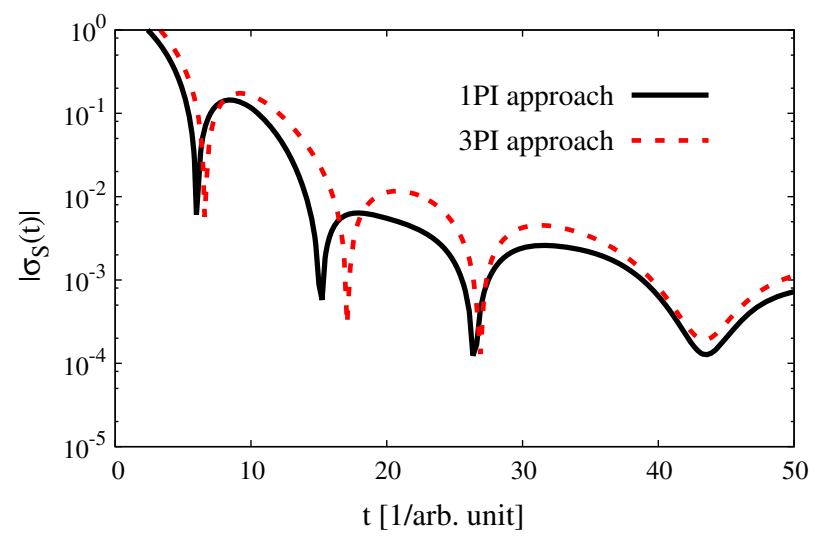

FIG. 10. Absolute value of the dressing function of Eq. (10), in $1 \mathrm{PI}$ and 3PI formulations. Cusps indicate zero crossings in $\sigma_{S}(t)$. 
the rainbow approach is merely a truncation artifact. The negative norm contributions to $\sigma_{S}(t)$ vanish upon the insertion of some additional tensor structures in the quarkgluon vertex. Although the here reported results are much more robust the observed positivity violation might still be an artifact of the approximate treatment of the fermion-gauge boson vertex. We are nevertheless confident that the truncation errors for the fermion propagator and the fermion-gauge boson are small enough to have no substantial impact on the bound-state spectra.

To summarize this subsection, it is encouraging that the final results with both one-loop diagrams included are almost insensitive to whether the 1PI or 3PI framework has been chosen. Since the additional vertex dressings in the 3PI version can be seen as an approximation to the effective re-summation of certain two-loop terms this leaves the possibility that the impact of the neglected terms is not overwhelmingly large, and that by neglecting them we have not made an error of qualitative importance. However, it should be pointed out that the aforementioned cancelation between the dressed three-gauge boson and fermion-gauge boson vertex in the 3PI formalism is almost certainly restricted to an $\mathrm{SU}(2)$ gauge theory. Given the change in color factors such a cancelation will not be present to such a high degree in a QCD calculation. Noting that the nonAbelian diagram carries a factor $N_{c}$ and the Abelian one is suppressed by a factor of $1 / N_{c}$ one can predict an even smaller impact of the Abelian diagram in QCD.

Finally, concerning the overall reliability of evaluations for certain propagators and vertices employed here, we mention that our results for dynamical fermion masses in Fig. 9 agree, on a qualitative level, with corresponding lattice estimates for $\mathrm{SU}(3)$ (see e.g., $[104,105]$ ) and $\mathrm{SU}(2)$ (see e.g., [106]) gauge field theories. However, lattice calculations cannot yet probe so deep into the infrared, meaning that the IR plateau for $M(p)$ is less pronounced in Monte Carlo simulations than in our investigation. Besides reproducing the shape of the function $M(p)$ reasonably well, compared to lattice results, we also see a trend which has already been observed on the lattice, regarding the infrared values of $M(p)$. Namely, in our $\mathrm{SU}(2)$ calculations the function $M(p)$ flattens out at lower values (in the respective internal units) in the IR, than it does in SU(3) computations with similar approximations; see e.g., [51]. In other words, we see a lower "constituent fermion mass" for an $\mathrm{SU}(2)$ case than an $\mathrm{SU}(3)$ one. Such a behavior has already been noted in lattice calculations; compare e.g., [104] with [106] (bearing in mind that some caution should be taken with such comparisons, due to differing lattice numerical setups). These facts give us confidence that our truncations, while possibly neglecting some effects of mild quantitative importance, are qualitatively robust enough to serve as a useful guide for future studies of this kind.

\section{E. Truncations for the kernel of the bound-state equations}

Besides the propagators and vertex functions discussed above the most important ingredient into the reported calculation is the symmetry-preserving BS kernel as obtained with the cutting technique of Eq. (6). For the truncated fermion-gauge boson vertex DS equation shown in Fig. 5 the corresponding truncated kernel is displayed in Fig. 11. In the 3PI-based truncation, there are additional dressings for the three-gauge boson and fermion-gauge boson vertices. As it is straightforward to implement them we are refraining from showing them explicitly.

\section{GROUND STATE MESONS}

Although our study is motivated, in large part, by exploring possibilities for a theory of beyond-thestandard-model physics we will mostly not concern ourselves with the composite Higgs and/or technicolor aspects of the considered gauge theory. The aim here is to obtain the ground state spectrum of $J \leq 1$ bound states and compare them with lattice results of Refs. [73,74]. In addition, to allow for a further development of truncations, we are interested in how the calculated ground state spectrum is influenced by the different contributions in the DS equations, and hereby especially in the DS equation

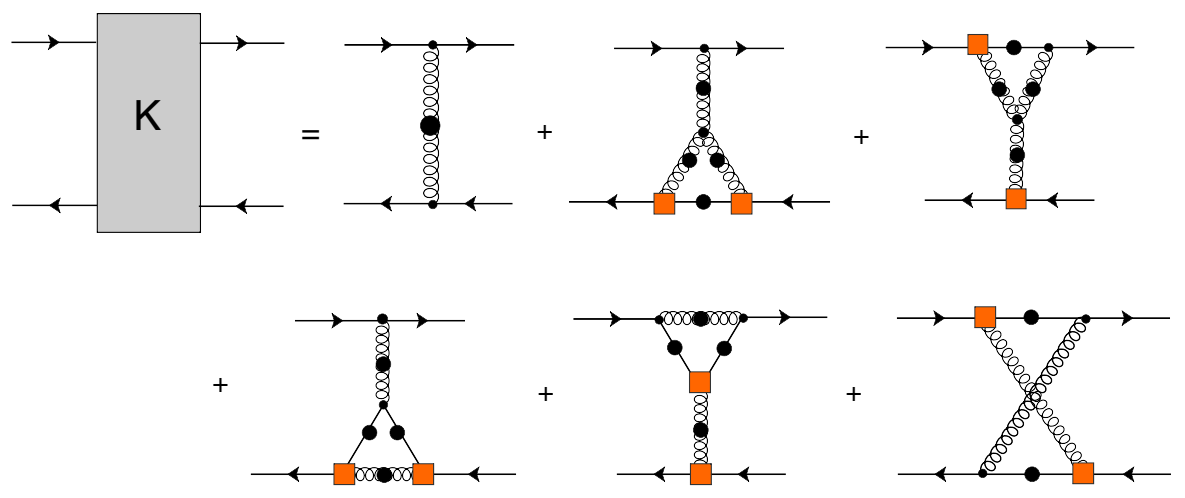

FIG. 11. A symmetry-preserving truncated BS kernel in agreement with the truncated fermion-gauge boson vertex DS equation in Fig. 5. 
TABLE I. Ground state masses in various truncations of the employed DS and BS equations in internal units: "NA" stands for the non-Abelian diagram only, "AB" for including the Abelian one. The given errors are purely numerical and are estimated within the employed extrapolation procedure, see text for details.

\begin{tabular}{lcccc}
\hline \hline$m_{J^{P C}}$ & $\mathrm{NA}, 1 \mathrm{PI}$ & $\mathrm{NA}+\mathrm{AB}, 1 \mathrm{PI}$ & $\mathrm{NA}, 3 \mathrm{PI}$ & $\mathrm{NA}+\mathrm{AB}, 3 \mathrm{PI}$ \\
\hline$m_{0^{-+}}$ & 0 & 0 & 0 & 0 \\
$m_{0^{++}}$ & $385(8)$ & $358(7)$ & $335(7)$ & $356(7)$ \\
$m_{1^{--}}$ & $628(13)$ & $583(12)$ & $597(12)$ & $567(11)$ \\
$m_{1^{++}}$ & $794(15)$ & $775(14)$ & $778(14)$ & $760(14)$ \\
$f_{\pi}$ & 68 & 72 & 62 & 70 \\
\hline \hline
\end{tabular}

for the fermion-gauge boson vertex. Of course, such tests can be performed completely with arbitrary internal units only. Therefore, we will choose a scale more for the matter of convenience than for necessity. The quantity which is fixed in scale-setting is the Higgs vacuum expectation value, $v_{e w}=246 \mathrm{GeV}$. On a purely formal level, this quantity is identical to the "pion" decay constant, i.e., the decay constant of the pseudoscalar Goldstone fields. It is then calculated via the identity [107]

$$
f_{P S}=\frac{Z_{2} N_{c}}{\sqrt{2} P^{2}} \operatorname{tr} \int_{k} \Gamma_{P S}(k,-P) S\left(k_{+}\right) \gamma_{5} P S\left(k_{-}\right),
$$

with $k_{ \pm}=k \pm P / 2$, and $\Gamma_{\pi}$ being the Goldstone boson BS amplitude, normalized with the Nakanishi condition [108]. (N.B.: In the above relation, we employ conventions for which $f_{\pi}=93 \mathrm{MeV}$ in QCD.)

The bound state masses in various truncations for the employed DS and BS equations are shown in Table I. As only the chiral limit is considered, the pseudoscalar, i.e., Goldstone boson, states are strictly massless. The results are displayed in arbitrary units in order to separate the direct influence of the truncations from the scale setting procedure. We provide the numerical value of $f_{\pi}$ in the last row of the respective columns to allow for transformation to physical scales. The errors on the masses are purely numerical and stem from the chosen way to extract the masses. In Euclidean field theory, the bound state on-shell condition $P^{2}=-M^{2}$ (with $M$ being the bound state mass) implies working with complex-valued total momentum $P$. However, there are ways to reliably estimate some of the hadronic observables by extrapolating from the region of spacelike $P^{2}$; see, e.g., Refs. [109-111] and references therein. Here we employ the inverse vertex extrapolation technique, which is explained in detail in [109]. Errors for some of the meson masses in Table I come from this approximate treatment. The efficiency of the method was thoroughly tested in [50], and it was found to be very reliable, at least for relatively light $J \leq 1$ mesons which we consider here. Note that, in the chiral limit, the Goldstone boson decay constant $f_{P S}$ is one of the very few quantities which can be calculated exactly purely from spacelike momenta or, more precisely, for $P^{2} \geq 0$. For this reason, the scale setting procedure does not introduce any additional uncertainties.

Let us take the results in the "NA, 1PI" column of Table I as the point of reference. Comparison with other truncations shows that all the modifications (addition of Abelian loops, the 3PI vertex dressings), induce moderate corrections. In terms of relative mass differences, the $0^{++}$channel seems to be most susceptible to various approximations, whereas the vector mesons (especially the axial one) are somewhat robust in this regard. The Abelian diagram induce modest relative changes to the bound state masses, ranging from $5 \%$ to $10 \%$ across different channels. Note that not only for QCD but also for all larger gauge groups (which have also been investigated with respect to beyondthe-standard-model physics; see, e.g., Ref. [112] and references therein) the impact of these diagrams would be further suppressed by group-theoretical factors.

It is interesting to note that the mass of the scalar in this calculation seems to be only mildly influenced by BRL effects. In the 1PI-based approach (including the Abelian diagram), one has $m_{0^{++}} / f_{P S}=5.0 \pm 0.1$, and for the 3PI one, $m_{0^{++}} / f_{P S}=5.1 \pm 0.1$; i.e., both values are very close to the RL result [50]. This is in contrast to the calculation of the scalar meson mass in Ref. [53]: The RL value $m_{0^{++}} / f_{\pi}=6.96$ is significantly lower than the obtained value in the much more sophisticated 3PI truncation employed there, $m_{0^{++}} / f_{\pi}=10.5 \pm 1.0$. Therefore, this comparison provides evidence that (i) the bound-state masses for an $\mathrm{SU}(3)$ gauge theory are much larger than for a SU(2) one (N. B.: This result is in agreement with the analysis of dynamically generated fermion masses in Ref. [112]), and (ii) BRL effects in this channel are more pronounced for SU(3) than SU(2). Having a look at the vector and axialvector channels these differences seems to be much smaller. The QCD study of Ref. [53] provides for the 3PI-3-loop truncation $m_{1^{--}} / f_{\pi}=7.0$ whereas we obtain $m_{1^{--}} / f_{P S}=8.1 \pm 0.2$. As usual BRL effects are small for the vector channel; cf. the discussion in the review [7] where the presence/absence of BRL corrections is related to importance/insignificance of spin-flip-type interactions among the constituents. For the axial vector channel BRL effects are significant, and the SU(3) versus $S U(2)$ comparison points towards an even smaller difference or maybe even almost no difference within the numerical accuracy, $m_{1^{++}} / f_{\pi}=12.4 \pm 1.0$ versus $m_{1^{++}} / f_{P S}=10.9 \pm 0.2$

The differences discussed above are important for future investigations which aim at a good numerical precision. Certainly, more work is needed to judge whether a level of "apparent convergence" has been reached already. From a qualitative perspective, however, one can note already some interesting trends. The ground state spectrum of the theory investigated here has also been studied on the lattice $[73,74]$, but as it is shown in Table II, the corresponding 
TABLE II. Light meson masses in various truncations of the quark-gluon DSE and the meson BSE, compared with the lattice data. All results are in units of TeV.

\begin{tabular}{lccccc}
\hline \hline & \multicolumn{3}{c}{$\mathrm{NA}+\mathrm{AB}}$, & $\mathrm{NA}+\mathrm{AB}$, & Lattice \\
$J^{P C}$ & $\mathrm{NA}, 1 \mathrm{PI}$ & 1PI & $\mathrm{NA}, 3 \mathrm{PI}$ & 3PI & {$[73,74]$} \\
\hline $0^{-+}$ & 0 & 0 & 0 & 0 & $\ldots$ \\
$0^{++}$ & $1.39(3)$ & $1.22(2)$ & $1.33(3)$ & $1.25(2)$ & $4.7 \pm 2.7$ \\
$1^{--}$ & $2.27(5)$ & $2.00(4)$ & $2.37(5)$ & $1.99(4)$ & $3.2 \pm 0.5$ \\
$1^{++}$ & $2.87(5)$ & $2.65(5)$ & $3.09(6)$ & $2.67(5)$ & $3.6 \pm 0.9$ \\
\hline \hline
\end{tabular}

results have relatively large uncertainties, definitely larger ones than in between our different truncations. It is interesting to note that the masses as obtained on the lattice seem to be systematically larger than our results. As a rule of thumb one may summarize the comparison by the statement that the masses from the DS equations are located at the lower end of the 1- $\sigma$-band of the lattice results.

An inspection of Table I reveals that all of the improvements of the simplest "1PI, NA" scenario which we have considered suppress the masses further. Taken together with the influence of pion backreaction, as investigated in [81], one is drawn towards the conclusion that all of the one-loop corrections to the fermion-gauge boson vertex beyond the simplest non-Abelian treatment invariably have a screening effect on the results for bound states, seemingly pushing them away from experimental data in QCD, or central lattice estimates in an SU(2) technicolor theory. This motivates than a fully self-consistent calculation for different gauge groups, especially because the meson mass results of Ref. [53] already show quite some improvement in this regard.

\section{CONCLUSIONS}

Building on the established knowledge of correlation functions in gauge theories in Landau gauge, we have studied the effects of various truncations of the DS equations for the fermion-gauge boson vertex, the fermion propagator, and the ground state spectrum in an SU(2) gauge theory. For both, the fundamental vertex functions and the bound state masses, we found relatively mild changes in results for different truncations. For the masses, they were on the order of roughly $5 \%$ to $10 \%$. Where the recent respective lattice results $[73,74]$ seem to indicate a significant difference to results in an $\mathrm{SU}(3)$ gauge theory, we obtained masses which were consistently lower than the central values of the lattice results but are nevertheless in agreement with them at an approximately $1-\sigma$ level. Therefore, differences to the $\mathrm{SU}(3)$ meson spectrum remain, especially for the scalar, but they are not as pronounced as indicated by the (central values of the) lattice results.

It remains to be seen if the methods outlined here can lead to certain improvements when applied to the baryon sector of QCD, for instance in the description of the nucleons' negative parity partner [51]. Also, the fact that even in our simplified framework the influence of the Abelian diagram in the fermion-gauge boson equation was found to be modest but still noticeable, is suggestive that in a self-consistent calculation these terms might induce potentially significant corrections because in the BS kernel there are then four fully dressed fermion-gauge boson vertices $[52,53]$.

For completeness, we wish also to address the issue on how some of our results might change if one considers gauge groups different from $\mathrm{SU}(2)$. We mentioned at the end of Sec. II D that we get a smaller constituent fermion mass, expressed in respective internal units, for an $\mathrm{SU}(2)$ gauge theory than an $\mathrm{SU}(3)$ one. This, in turn, translates to smaller meson masses, in units of the "pion" decay constant $f_{\pi}$, in the two-colored setup compared to a three-colored theory, see e.g., [51]. This scaling behavior for masses arises naturally from the color Casimir operator $C_{f}$ in Eq. (4), which makes the fermion self-energy more relevant as the number of colors is increased. Naively, we would expect that all hadrons become more massive as more colors are added to the theory. This expectation is in accord with the main conclusions of [112]. However, in all these considerations it is implicitly assumed that the backcoupling of fermions onto the Yang-Mills sector has only a mild quantitative impact. Based on some current lattice results for an SU(3) gauge theory (e.g., [113]), such an assumption seems justified for a relatively small number of light, fundamentally-charged fermions, but it might not hold if the number of light flavors is increased [83], or if one looks at fermions belonging to higher-dimensional representations of the gauge group [4]. For the latter scenarios, a careful investigation on how fermions influence the Yang-Mills sector should be undertaken, which is beyond the scope of our current model. We are presently working on improved approximations to our systems of equations, which would allow one to assess the influence of fermions in a reliable and qualitatively robust way.

In summary, the investigation presented here has provided one further step towards a fully self-consistent treatment of gauge-invariant bound states in gauge theories with a sufficiently sophisticated truncation scheme of DS and BS equations. Establishing such a scheme will first of all provide more insight into the binding mechanisms for highly relativistic bound states. For theories with a walking behavior of the coupling (which implies that one deals with a multiscale problem), functional methods based on continuum quantum field theories may offer even a higher precision than lattice calculations.

\section{ACKNOWLEDGMENTS}

M. V. gratefully acknowledges financial support by the University of Graz and by the Austrian science fund FWF via the Doctoral Program W1203 and the Schrödinger 
Grant No. J3854-N36. We also thank Hèlios SanchisAlepuz for his critical reading of this manuscript and his comments.

\section{APPENDIX: NUMERICAL METHOD FOR THE CALCULATION OF THE FERMION-GAUGE BOSON VERTEX}

In the treatment of the fermion-gauge boson vertex, we implement a scheme described in Ref. [49]. The main idea is to use on the rhs of the vertex equation a projection of the vertex on its tree-level tensor structure and therefore significantly simplified internal vertices. This projection is achieved by constructing an effective dressing function $\lambda\left(k^{2}\right)$; cf. Eq. (7). To be explicit, the following parametrization is used,

$$
\begin{aligned}
\lambda\left(k^{2}\right)= & h Z_{1 f}\left\{\frac{L\left(M_{0}\right)}{1+y}+\frac{1}{1+z}\right. \\
& \left.\times\left[\frac{4 \pi}{\beta_{0} \alpha_{\mu}}\left(\frac{1}{\log (x)}-\frac{1}{1-x}\right)\right]^{18 / 44}\right\},
\end{aligned}
$$

with $h=2.302, x=k^{2} / 0.6, y=k^{2} / 0.34, z=k^{2} / 0.33$, $\beta_{0}=11 N_{c} / 3$, and $\alpha_{\mu}=1.114$ the renormalized coupling at a scale $\mu=3$ (in arbitrary units). The infrared enhancement $L\left(M_{0}\right)$ depends on the quark mass at zero momentum $\left(M_{0}=M\left(p^{2}\right)=0\right)$ and is parametrized as a ratio of polynomials:

$$
L\left(M_{0}\right)=\frac{a+b M_{0}+c M_{0}^{2}}{M_{0}+d M_{0}^{2}} .
$$

The coefficients $a, b, c$ and $d$ are determined such that the total model dressing $\lambda\left(k^{2}\right)$ fits reasonably precisely the treelevel projection of the full calculated fermion-gauge boson
TABLE III. Coefficients of Eq. (A2) in various approximations for the fermion-gauge boson vertex. (N. B.: The "NA + AB, $1 \mathrm{PI}$ " parameters have the same numerical values as ones of "NA, 1PI".)

\begin{tabular}{lccc}
\hline \hline Coeff. & NA, 1PI & NA, 3PI & NA + AB, 3PI \\
\hline$a$ & 0.244 & -0.014 & 0.342 \\
$b$ & 1.788 & 3.20 & 1.037 \\
$c$ & -0.198 & 0.842 & -0.812 \\
$d$ & 0.293 & 1.928 & -0.621 \\
\hline \hline
\end{tabular}

vertex. These parameters are given in Table III for the truncations considered in this work.

For completeness we provide the covariant tensor decomposition for the full calculated vertex $\Gamma^{\mu}\left(p_{1}, p_{2}\right)$, with $p_{1}$ and $p_{2}$ denoting, respectively, the incoming and outgoing fermion momenta. Defining the relative momentum $l=\left(p_{1}+p_{2}\right) / 2$, and the outgoing gluon momentum $k=p_{2}-p_{1}$, we use the orthonormal combinations:

$$
\begin{aligned}
t^{\mu} & =\hat{k}^{\mu}, \\
s^{\mu} & =\hat{h}^{\mu} \quad \text { with } \quad h^{\mu}=T_{\alpha \beta}^{(t)} l^{\beta}, \\
\gamma_{T T}^{\mu} & =T^{(t) \mu \alpha} T_{\alpha \nu}^{(s)} \gamma^{\nu}=\gamma^{\mu}-\not t^{\mu}-\not s^{\mu},
\end{aligned}
$$

with the hat denoting normalization of the corresponding four-vector. Any components proportional to $t^{\mu}$ will be projected out in Landau gauge, leaving eight purely transverse tensors. Their basis is chosen to be

$$
\left(\gamma_{T T}^{\mu}, s^{\mu}\right) \times(\mathbf{1}, \phi, t, \phi t) .
$$

Here, 1 stands for a Dirac unity matrix. The $T_{1}$ dressing function, plotted in the right panel of Fig. 9, is the prefactor of the tensor $\gamma_{T T}$.
[1] D. B. Kaplan, H. Georgi, and S. Dimopoulos, Phys. Lett. 136B, 187 (1984).

[2] K. Agashe, R. Contino, and A. Pomarol, Nucl. Phys. B719, 165 (2005).

[3] E. Farhi and L. Susskind, Phys. Rep. 74, 277 (1981).

[4] J. R. Andersen et al., Eur. Phys. J. Plus 126, 81 (2011).

[5] Y. Hochberg, E. Kuflik, T. Volansky, and J. G. Wacker, Phys. Rev. Lett. 113, 171301 (2014); Y. Hochberg, E. Kuflik, H. Murayama, T. Volansky, and J. G. Wacker, Phys. Rev. Lett. 115, 021301 (2015); Y. Hochberg, E. Kuflik, and H. Murayama, J. High Energy Phys. 05 (2016) 090.

[6] C. B. Lang, AIP Conf. Proc. 1735, 020002 (2016).

[7] G. Eichmann, H. Sanchis-Alepuz, R. Williams, R. Alkofer, and C. S. Fischer, Prog. Part. Nucl. Phys. 91, 1 (2016).
[8] H. A. Bethe and E. E. Salpeter, Phys. Rev. 82, 309 (1951); E. E. Salpeter and H. A. Bethe, Phys. Rev. 84, 1232 (1951).

[9] F. J. Dyson, Phys. Rev. 75 (1949) 1736.

[10] J. S. Schwinger, Proc. Natl. Acad. Sci. U.S.A. 37, 452 (1951).

[11] C. Wetterich, Phys. Lett. B 301, 90 (1993).

[12] T. R. Morris, Int. J. Mod. Phys. A 09, 2411 (1994).

[13] C. D. Roberts and A. G. Williams, Prog. Part. Nucl. Phys. 33, 477 (1994).

[14] R. Alkofer and L. von Smekal, Phys. Rep. 353, 281 (2001).

[15] C. S. Fischer, J. Phys. G 32, R253 (2006).

[16] I. C. Cloet and C. D. Roberts, Prog. Part. Nucl. Phys. 77, 1 (2014).

[17] J. Berges, N. Tetradis, and C. Wetterich, Phys. Rep. 363, 223 (2002). 
[18] J. M. Pawlowski, Ann. Phys. (Amsterdam) 322, 2831 (2007).

[19] P. Maris, C. D. Roberts, and P. C. Tandy, Phys. Lett. B 420, 267 (1998).

[20] P. Maris and C. D. Roberts, Phys. Rev. C 56, 3369 (1997).

[21] P. Maris and P. C. Tandy, Phys. Rev. C 60, 055214 (1999).

[22] A. Krassnigg, Proc. Sci., CONFINEMENT8 (2008) 075 [arXiv:0812.3073].

[23] A. Krassnigg, Phys. Rev. D 80, 114010 (2009).

[24] M. Blank, A. Krassnigg, and A. Maas, Phys. Rev. D 83, 034020 (2011).

[25] A. Krassnigg and M. Blank, Phys. Rev. D 83, 096006 (2011).

[26] M. Blank and A. Krassnigg, Phys. Rev. D 84, 096014 (2011).

[27] H. L. L. Roberts, L. Chang, I. C. Cloet, and C. D. Roberts, Few-Body Syst. 51, 1 (2011).

[28] C. S. Fischer, S. Kubrak, and R. Williams, Eur. Phys. J. A 50, 126 (2014).

[29] T. Hilger, C. Popovici, M. Gomez-Rocha, and A. Krassnigg, Phys. Rev. D 91, 034013 (2015).

[30] C. S. Fischer, S. Kubrak, and R. Williams, Eur. Phys. J. A 51, 10 (2015).

[31] T. Hilger, M. Gomez-Rocha, and A. Krassnigg, Phys. Rev. D 91, 114004 (2015).

[32] G. Eichmann, R. Alkofer, A. Krassnigg, and D. Nicmorus, Phys. Rev. Lett. 104, 201601 (2010).

[33] D. Nicmorus, G. Eichmann, and R. Alkofer, Phys. Rev. D 82, 114017 (2010).

[34] H. Sanchis-Alepuz, R. Alkofer, G. Eichmann, and R. Williams, Proc. Sci., QCD-TNT-II2011 (2011) 041 [arXiv: 1112.3214].

[35] G. Eichmann, Phys. Rev. D 84, 014014 (2011).

[36] H. Sanchis-Alepuz, R. Williams, and R. Alkofer, Phys. Rev. D 87, 096015 (2013).

[37] H. Sanchis-Alepuz and C. S. Fischer, Eur. Phys. J. A 52, 34 (2016).

[38] H. Sanchis-Alepuz and R. Williams, Comput. Phys. Commun. 232, 1 (2018).

[39] H. J. Munczek, Phys. Rev. D 52, 4736 (1995).

[40] A. Bender, C. D. Roberts, and L. Von Smekal, Phys. Lett. B 380, 7 (1996).

[41] A. Bender, W. Detmold, C. D. Roberts, and A. W. Thomas, Phys. Rev. C 65, 065203 (2002).

[42] P. Watson, W. Cassing, and P. C. Tandy, Few-Body Syst. 35, 129 (2004).

[43] P. Watson and W. Cassing, Few-Body Syst. 35, 99 (2004).

[44] M. S. Bhagwat, A. Holl, A. Krassnigg, C. D. Roberts, and P. C. Tandy, Phys. Rev. C 70, 035205 (2004).

[45] H. H. Matevosyan, A. W. Thomas, and P. C. Tandy, Phys. Rev. C 75, 045201 (2007).

[46] C. S. Fischer and R. Williams, Phys. Rev. Lett. 103, 122001 (2009).

[47] A. Windisch, M. Hopfer, and R. Alkofer, Acta Phys. Pol. B Proc. Suppl. 6, 347 (2013).

[48] M. Gomez-Rocha, T. Hilger, and A. Krassnigg, Few-Body Syst. 56, 475 (2015).

[49] R. Williams, Eur. Phys. J. A 51, 57 (2015).

[50] M. Vujinovic and R. Williams, Eur. Phys. J. C 75, 100 (2015).
[51] H. Sanchis-Alepuz and R. Williams, Phys. Lett. B 749, 592 (2015).

[52] H. Sanchis-Alepuz and R. Williams, J. Phys. Conf. Ser. 631, 012064 (2015).

[53] R. Williams, C. S. Fischer, and W. Heupel, Phys. Rev. D 93, 034026 (2016).

[54] C. S. Fischer, P. Watson, and W. Cassing, Phys. Rev. D 72, 094025 (2005).

[55] C. S. Fischer, D. Nickel, and J. Wambach, Phys. Rev. D 76, 094009 (2007).

[56] C. S. Fischer, D. Nickel, and R. Williams, Eur. Phys. J. C 60, 47 (2009).

[57] C. S. Fischer and R. Williams, Phys. Rev. D 78, 074006 (2008).

[58] L. Chang and C. D. Roberts, Phys. Rev. Lett. 103, 081601 (2009).

[59] L. Chang and C. D. Roberts, Phys. Rev. C 85, 052201 (2012).

[60] W. Heupel, T. Goecke, and C. S. Fischer, Eur. Phys. J. A 50, 85 (2014)

[61] A. K. Cyrol, M. Mitter, J. M. Pawlowski, and N. Strodthoff, Phys. Rev. D 97, 054006 (2018); A. K. Cyrol, L. Fister, M. Mitter, J. M. Pawlowski, and N. Strodthoff, Phys. Rev. D 94, 054005 (2016).

[62] R. Alkofer, A. Maas, W. Mian, M. Mitter, J. París-Lopez, J. Pawlowski, and N. Wink, arXiv:1810.07955; J. ParísLopez, R. Alkofer, A. Maas, W. Mian, M. Mitter, J. Pawlowski, and H. Sanchis-Alepuz, J. Phys. Conf. Ser. 1024, 012009 (2018).

[63] S. Hands, J. B. Kogut, M. P. Lombardo, and S. E. Morrison, Nucl. Phys. B558, 327 (1999).

[64] R. Aloisio, V. Azcoiti, G. Di Carlo, A. Galante, and A. F. Grillo, Nucl. Phys. B606, 322 (2001).

[65] S. Hands, I. Montvay, S. Morrison, M. Oevers, L. Scorzato, and J. Skullerud, Eur. Phys. J. C 17, 285 (2000).

[66] J. B. Kogut, D. K. Sinclair, S. J. Hands, and S. E. Morrison, Phys. Rev. D 64, 094505 (2001).

[67] S. Muroya, A. Nakamura, and C. Nonaka, Phys. Lett. B 551, 305 (2003).

[68] S. Hands, S. Kim, and J. I. Skullerud, Eur. Phys. J. C 48, 193 (2006).

[69] S. Cotter, P. Giudice, S. Hands, and J. I. Skullerud, Phys. Rev. D 87, 034507 (2013).

[70] B. Bahrampour, B. Wellegehausen, and L. von Smekal, Proc. Sci., LATTICE2016 (2016) 070 [arXiv:1612.00285].

[71] N. Khan, J. M. Pawlowski, F. Rennecke, and M. M. Scherer, arXiv:1512.03673.

[72] R. Contant and M. Q. Huber, Phys. Rev. D 96, 074002 (2017).

[73] R. Arthur, V. Drach, M. Hansen, A. Hietanen, C. Pica, and F. Sannino, Phys. Rev. D 94, 094507 (2016).

[74] R. Arthur, V. Drach, A. Hietanen, C. Pica, and F. Sannino, arXiv:1607.06654.

[75] V. Drach, T. Janowski, C. Pica, J. Rantaharju, and F. Sannino, Proc. Sci., LATTICE2016 (2017) 229.

[76] V. Drach, T. Janowski, and C. Pica, Eur. Phys. J. Web Conf. 175, 08020 (2018).

[77] J. W. Lee, B. Lucini, and M. Piai, J. High Energy Phys. 04 (2017) 036. 
[78] G. Cacciapaglia and F. Sannino, J. High Energy Phys. 04 (2014) 111.

[79] R. Alkofer and H. Reinhardt, Lect. Notes Phys., M: Monogr. 33, 1 (1995).

[80] R. Foadi, M. T. Frandsen, and F. Sannino, Phys. Rev. D 87, 095001 (2013).

[81] R. Williams, Eur. Phys. J. Web Conf. 3, 03005 (2010).

[82] H. Sanchis-Alepuz, C. S. Fischer, and S. Kubrak, Phys. Lett. B 733, 151 (2014).

[83] M. Hopfer, C. S. Fischer, and R. Alkofer, J. High Energy Phys. 11 (2014) 035; Acta Phys. Pol. B Proc. Suppl. 7, 613 (2014).

[84] R. Alkofer, C. S. Fischer, F. J. Llanes-Estrada, and K. Schwenzer, Ann. Phys. (Amsterdam) 324, 106 (2009).

[85] A. Windisch, Ph.D. thesis, University of Graz, 2014, http:// unipub.uni-graz.at/obvugrhs/download/pdf/242468.

[86] M. Hopfer, Ph.D. thesis, University of Graz, 2014, http:// unipub.uni-graz.at/obvugrhs/download/pdf/269085.

[87] F. J. Llanes-Estrada, C. S. Fischer, and R. Alkofer, Nucl. Phys. B, Proc. Suppl. 152, 43 (2006).

[88] M. Hopfer, A. Windisch, and R. Alkofer, Proc. Sci., ConfinementX2012 (2012) 073 [arXiv:1301.3672].

[89] M. Peláez, M. Tissier, and N. Wschebor, Phys. Rev. D 92 , 045012 (2015).

[90] H. F. Fu and Q. Wang, Phys. Rev. D 93, 014013 (2016).

[91] E. Rojas, J. P. B. C. de Melo, B. El-Bennich, O. Oliveira, and T. Frederico, J. High Energy Phys. 10 (2013) 193.

[92] A. C. Aguilar, D. Binosi, D. Ibañez, and J. Papavassiliou, Phys. Rev. D 90, 065027 (2014).

[93] M. Mitter, J. M. Pawlowski, and N. Strodthoff, Phys. Rev. D 91, 054035 (2015).

[94] O. Oliveira, T. Frederico, W. de Paula, and J. P. B. C. de Melo, Eur. Phys. J. C 78, 553 (2018).

[95] J. Berges, Phys. Rev. D 70, 105010 (2004).
[96] M. Q. Huber, arXiv:1808.05227.

[97] G. Eichmann, R. Williams, R. Alkofer, and M. Vujinovic, Phys. Rev. D 89, 105014 (2014).

[98] A. Blum, M. Q. Huber, M. Mitter, and L. von Smekal, Phys. Rev. D 89, 061703 (2014).

[99] A. Cucchieri, A. Maas, and T. Mendes, Phys. Rev. D 77, 094510 (2008).

[100] M. Vujinovic and T. Mendes, arXiv:1807.03673.

[101] L. von Smekal, K. Maltman, and A. Sternbeck, Phys. Lett. B 681, 336 (2009).

[102] M. Hopfer and R. Alkofer, Acta Phys. Pol. B Proc. Suppl. 6, 929 (2013).

[103] R. Alkofer, W. Detmold, C. S. Fischer, and P. Maris, Phys. Rev. D 70, 014014 (2004).

[104] O. Oliveira, A. Kızılersu, P. J. Silva, J. I. Skullerud, A. Sternbeck, and A. G. Williams, Acta Phys. Pol. B Proc. Suppl. 9, 363 (2016).

[105] D. Trewartha, W. Kamleh, D. Leinweber, and D. S. Roberts, Phys. Rev. D 88, 034501 (2013).

[106] P. O. Bowman, K. Langfeld, D. B. Leinweber, A. O' Cais, A. Sternbeck, L. von Smekal, and A. G. Williams, Phys. Rev. D 78, 054509 (2008).

[107] P. C. Tandy, Prog. Part. Nucl. Phys. 39, 117 (1997).

[108] N. Nakanishi, Phys. Rev. 139, B1401 (1965).

[109] M. S. Bhagwat, A. Hoell, A. Krassnigg, C. D. Roberts, and S. V. Wright, Few-Body Syst. 40, 209 (2007).

[110] S. M. Dorkin, L. P. Kaptari, T. Hilger, and B. Kampfer, Phys. Rev. C 89, 034005 (2014).

[111] R. A. Tripolt, P. Gubler, M. Ulybyshe, and L. Von Smekal, arXiv:1801.10348.

[112] F. J. Llanes-Estrada and A. Salas-Bernárdez, arXiv: 1808.05102 .

[113] A. Ayala, A. Bashir, D. Binosi, M. Cristoforetti, and J. Rodriguez-Quintero, Phys. Rev. D 86, 074512 (2012). 